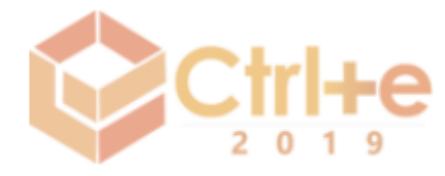

IV Congresso sobre Tecnologias na Educação (Ctrl+E 2019)

Recife, Pernambuco - Brasil

28 a 30 de agosto de 2019

\title{
Jogo Mobile como Ferramenta de Educação para Prevenção da Doença de Chagas
}

\author{
Lucio L. Oliveira ${ }^{1}$, Ellison Ribeiro ${ }^{1}$, Marcos Seruffo ${ }^{1}$, Dilma de Souza ${ }^{2}$, Tereza \\ Sanches $^{2}$ \\ ${ }^{1}$ Instituto de Tecnologia - Universidade Federal do Pará (UFPA) Caixa postal 479.PABX \\ +55 91 3201-7000 - Belém - PA - Brasil \\ ${ }^{2}$ Instituto de Ciência da Saúde - Universidade Federal do Pará (UFPA) Caixa postal \\ 479.PABX +55 91 3201-7000 - Belém - PA - Brasil

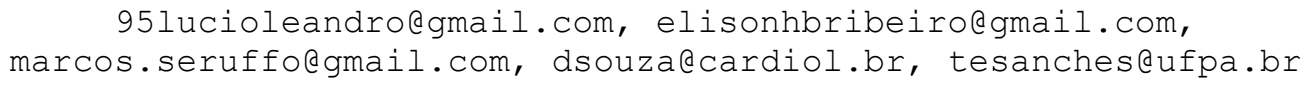

Abstract: Chagas disease (American trypanosomiasis) is an infectious tropical disease that is still neglected. Previously considered a rural endemic disease, it is becoming urban due to consumption, int cities, of food contaminated by the protozoan that causes the illness, especially in the state of Pará where people often consume açaí berries without a proper sanitation process. If the forms of contagion of a disease are well known, it becomes easier to prevent it, and education is crucial for that. This article proposes the creation of an educational and accessible game about the disease. By knowing that making players to engage in educational entertainment products is challenging, game design principles are taken into account to develop this experience.

Resumo: A doença de Chagas (Tripanossomíase americana) é uma doença tropical infecciosa que ainda é bastante negligenciada. A enfermidade, antes considerada rural, está se tornando uma endemia urbana devido ao consumo, nas cidades, de alimento contaminado pelo protozoário causador da doença, sobretudo no estado do Pará onde a população consome açaí sem higienização. Se as formas de contágio de uma doença são bem conhecidas, torna-se mais fácil preveni-la e, para tanto, educação é fundamental, por isso, este artigo propõe a criação de um jogo educativo e acessível sobre a doença de Chagas. Sabendo-se do desafio que é engajar usuários em produtos de entretenimento cujo foco é educação, princípios do game design são levados em conta no desenvolvimento da experiência.

\section{Introdução}

A doença de Chagas (Tripanossomíase americana) é uma doença infecciosa causada pelo protozoário Trypanossoma cruzi e se mantém com taxa constante de infectados até o ano atual (2019), contudo ainda é uma das doenças tropicais mais negligenciadas [Médicos sem 
Fronteiras (MSF), 2018], sendo que o estado do Pará é responsável por $83 \%$ dos casos no País [Ministério da Saúde, 2019]. Segundo a CONITEC (Comissão Nacional de Incorporação de Tecnologias no SUS), em 2016 estimou-se que na América Latina foram perdidos cerca de 752.000 dias de trabalho ao ano devido à doença de Chagas e mais de 1,2 bilhões de dólares por ano em sete países sul-americanos. A doença afeta principalmente o coração e a principal forma de transmissão é a falta de higiene com alimentos, seguida pela transmissão vetorial pelo inseto Triatoma infestans, chamado popularmente de "barbeiro".

Supõe-se que tais prejuízos causados pela doença de Chagas poderiam ser evitados com um trabalho de disseminação de informações focados na sua prevenção e um dos veículos dessa divulgação poderiam ser os aparelhos móveis. No Brasil, nos últimos três meses, das 120,7 milhões de pessoas que acessaram a internet, $49 \%$ o fizeram utilizando somente o celular, $4 \%$ somente o computador e $47 \%$ ambos [Comitê Gestor da Internet no Brasil, 2018]. E o mercado de games brasileiro cresce rapidamente ultrapassando públicos de cinema e teatro, arrecadando bilhões de dólares todos os anos. Somente na América Latina a estimativa de faturamento era de cerca de US\$ 134 bilhões em 2018 [ECommerce, 2018]. Sendo que, o mercado de games mobile corresponde a $41 \%$ do faturamento total no ano de 2018, enquanto, consoles (aparelhos convencionais de videogames) equivalem a $30 \%$ e de computadores, $29 \%$. Desta forma, soluções que possam aproveitar o legado já estabelecido pelo uso de smartphones, integrando ludicidade e educação, ganha forte relevância.

Dispondo-se deste contexto, é possível perceber a importância que o mercado de games e smartphones têm hoje no Brasil. Portanto, pode ser estimulável utilizar a tecnologia a favor da educação através de jogos mobile como ferramenta de ensino e aprendizagem, principalmente para crianças e adolescentes. Sabendo disso, este artigo tem o objetivo de analisar a eficácia da educação ludificada aplicada a cuidados com a saúde, foi desenvolvido um jogo, descrito neste artigo, que apresenta orientações sobre a doença de Chagas, visando a conscientizar a população através de uma forma mais criativas, ensinando formas de prevenção, contágio e tratamento da enfermidade

Em resumo, o trabalho realizado faz a integração de tecnologia móvel com o meio físico de comunicação, jogos como meio de disseminação de conhecimento e saúde como o foco das informações. Este se apresenta da seguinte forma: na seção 2 é mostrado o referencial teórico usado como base para criação do projeto; a seção 3 apresenta a metodologia utilizada para criação e configuração no desenvolvimento; na seção 4 abordase como ficou o resultado da versão beta (versão em desenvolvimento, mas já usável) do jogo em uma aplicação no celular; e a seção 5 discute o resultado do artigo realizado.

\section{Referencial Teórico}

É notável que a tecnologia revolucionou o modo de vida da sociedade desde a primeira revolução industrial, acelerando-o e intensificando-o em vários âmbitos. Hoje, alguns especialistas afirmam estar acontecendo a quarta revolução industrial, em que o mundo está sendo tomado pela evolução no campo de inteligência artificial, já que "estamos a bordo de uma revolução tecnológica que transformará fundamentalmente a forma como vivemos, trabalhamos e nos relacionamos. Em sua escala, alcance e complexidade, a transformação será diferente de qualquer coisa que o ser humano tenha experimentado antes" [Klaus 
Schwab, 2016].

Sabendo-se disso, é fundamental a aprimoração na maneira de educar desempenhada hoje no Brasil. Aproveitando a alta do mercado de entretenimento digital, um projeto que integre tecnologia com aprendizagem é extremamente oportuno para o aprimoramento de saúde e educação no Brasil também por causa da doença de Chagas, que tem pouca divulgação e ainda mantém uma alta taxa de transmissão, principalmente em áreas pobres da região norte do país. Dentre as doenças endêmicas, a doença de Chagas ocupa um lugar de destaque, seja pelo grau de morbidade e letalidade, seja pelo alto custo econômico e social que representa, tendo no Brasil a estimativa de 2 a 3 milhões de pessoas infectadas [Alana Costa \& Darlan Cândido, 2016].

Considerando esses números alarmantes, percebe-se a urgente necessidade de educar para prevenir e a eficácia da utilização de jogos na educação infanto-juvenil é um método já testado e aprovado, o que torna muito conveniente um aumento no desenvolvimento de projetos deste tipo, pois em grande parte apresenta resultado positivo, segundo alguns argumentos de outros autores: "a atividade educativa mediada pelo uso de um jogo contribuiu para a aquisição de conhecimento sobre amamentação e cuidados com o recém-nascido" [Fonseca, \& cols., 2002]; "enfrentamento de situações problemas nos jogos. Tais situações obrigam os jogadores a organizar novas estratégias de ação para alcançar um resultado favorável" [Cezar, \& cols., 2008]. Os jogos podem ainda apresentar algumas vantagens ou objetivos indiretos, como o desenvolvimento da memória visual e auditiva; a coordenação motora ampla e fina; podem proporcionar orientação temporal e espacial em duas e três dimensões [Liliana Passerino, 2015].

\section{Metodologia}

Para a elaboração da estratégia educativa, foi desenvolvido um projeto multidisciplinar envolvendo saúde e educação tendo como requisito um estudo à parte sobre a doença de Chagas, como seus mecanismos de transmissão, sintomas e tratamentos; para isso fez-se necessário o uso de programas de edição de imagem (InkScape e Photofiltre), edição de som (Audacity) e principalmente a plataforma onde tudo foi integrado em forma de jogo digital, o Unity 2018.3. A partir do levantamento bibliográfico e do uso dos softwares citados, foi possível a criação de um jogo mobile, disponibilizado para Android. Foram considerados e aplicados diversos recursos capazes de fornecer mais realismo físico à aplicação, por exemplo, conceitos de atrito, força gravitacional e princípios de movimento aplicados nos personagens e nos objetos pelo cenário.

Toda a programação do jogo é realizada principalmente na linguagem C\#, mas também sendo possível alterar para C++. No projeto descrito neste artigo foi utilizado C\# e o Visual Studio como editor dos algoritmos, software igualmente disponibilizado de forma gratuita e com compatibilidade aos outros programas. A Unity® também oferece gratuitamente uma vasta gama de ferramentas dentro do seu próprio software, sendo possível controlar animação, física, fluxo do jogo e de telas além de uma organização simples, exigindo como requisito basicamente somente um pouco de conhecimento em programação, sendo de fácil organização na tela, mostrando a cena ao programador, como ficará no celular e as pastas e propriedades dos arquivos criados. O fluxo do jogo funciona da seguinte forma se segue como é mostrado no diagrama abaixo. 

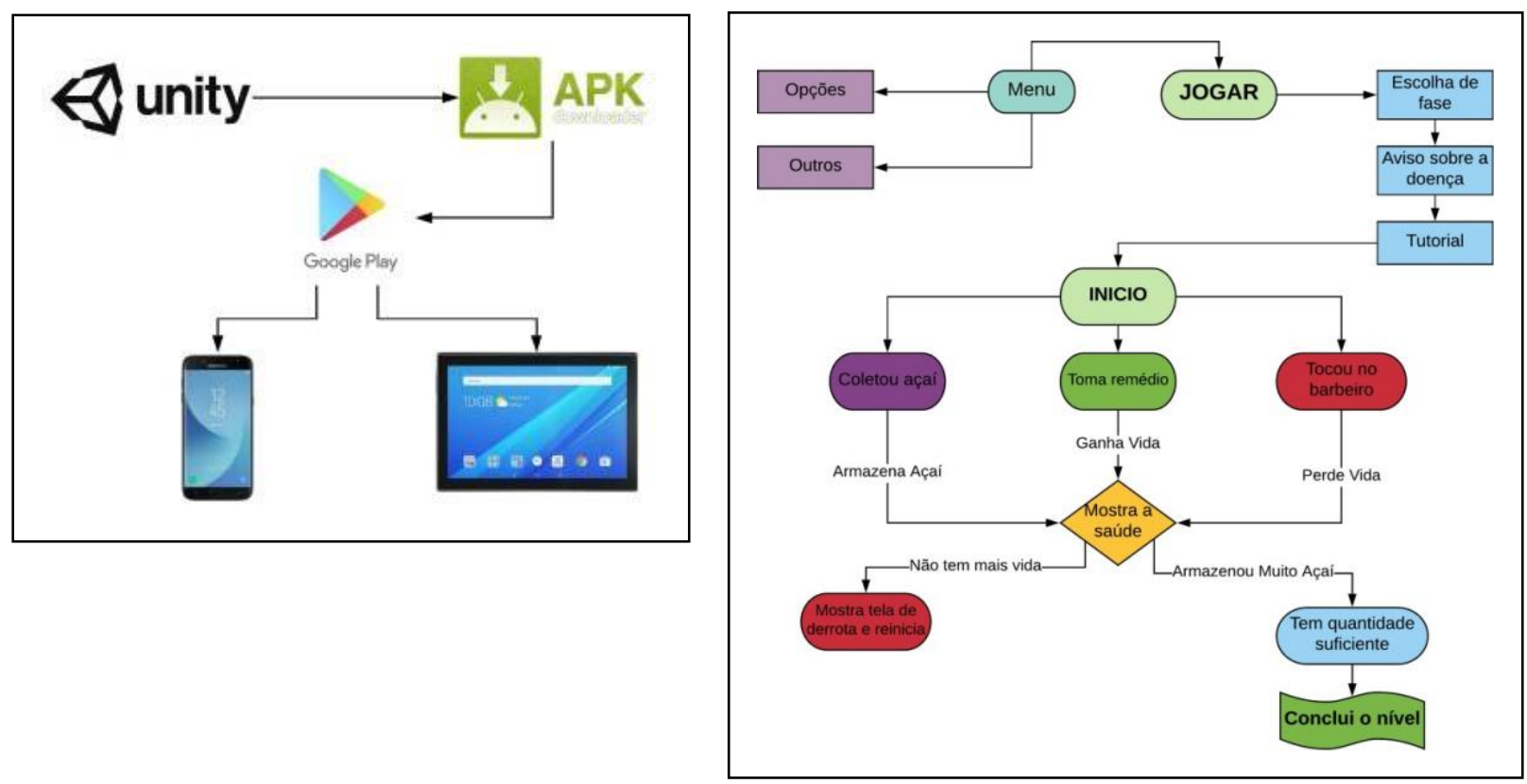

Figura 1. Forma de distribuição do aplicativo.

Figura 2. Fluxo do jogo.

\section{Resultados}

Ao final são apresentadas as telas obtidas com o desenvolvimento do jogo, pretendendo alcançar um grande público infanto-juvenil, visando, principalmente, moradores da região norte do país, devido à maior taxa de contágio, com o intuito de mostrar que a aplicação contém métodos didáticos e lúdicos que estimulam o aprendizado dos jogadores e também auxiliam a desenvolver uma visão crítica dos problemas sociais e de saneamento dos arredores da sua comunidade. Na Figura 3 apresenta-se o menu inicial, onde o jogador tem acesso às configurações do jogo e pode começar a jogar. Após iniciar será mostrado um aviso introdutório ao jogo, mostrado na Figura 4, além de outros avisos explicando a dinâmica da doença ao decorrer do jogo para auxiliar em como proceder durante cada etapa.

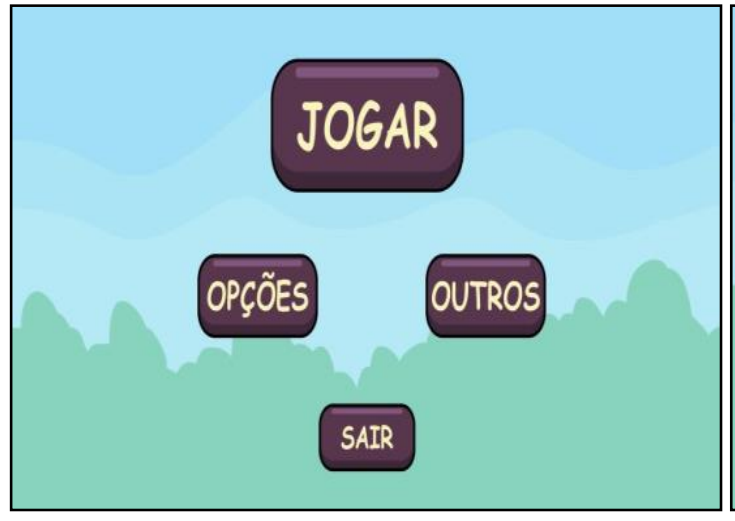

Figura 3. Menu inicial

\section{ChaGame}

Bem vindo(a) ao ChaGame. Aqui você aprenderá sobre a doença de Chagas enquanto se diverte!

Durante o jogo, desvie do barbeiro e colete açaí.

\section{INICIAR}

Após o aviso e o breve tutorial da jogabilidade do personagem, é possível começar a 
jogar, indo para frente, para trás e pulando (a partir do uso de setas direcionais) com intenção de coletar todos os frutos de açaí para passar de nível. O jogador começa com o status de "Saudável" e este se altera cada vez que entrar em contato com o barbeiro ou com açaí contaminado, mas melhorando a cada vez que coletar remédio, sendo ao final de cada fase entregue todos os açaís coletados para produzir um açaí de qualidade em um ponto de comercialização autorizado pela vigilância sanitária passando, então, de nível ao tomá-lo.

Ao decorrer do jogo, são adaptadas as formas em que o personagem se encontra dependendo de seu estado de saúde, como representado na Figura 6, simbolizando: saudável - ótimo estado de saúde, doente - contagiado, apresentando febre e dor no corpo, muito doente, apresentando arritmia cardíaca, extrema fadiga e dor de cabeça.

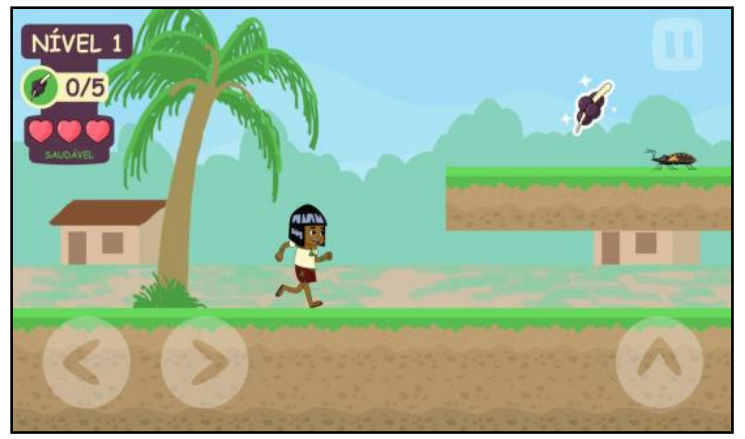

Figura 5. Resultado do jogo em funcionamento

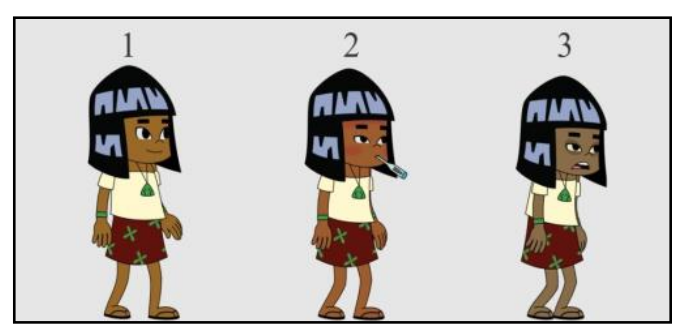

Figura 6. Estágios da doença

Em cada estágio acima será mostrada toda a descrição da doença de forma lúdica, sem atrapalhar o fluxo do jogo ou a jogabilidade do usuário, informando-o sobre cada mudança que haverá no corpo do infectado e como tratá-la da melhor forma. Caso o jogador não consiga se curar depois do estágio 3 e continue tomando açaí infectado, ele morrerá e aparecerá uma tela de derrota, constando a quantidade total de açaí coletado.

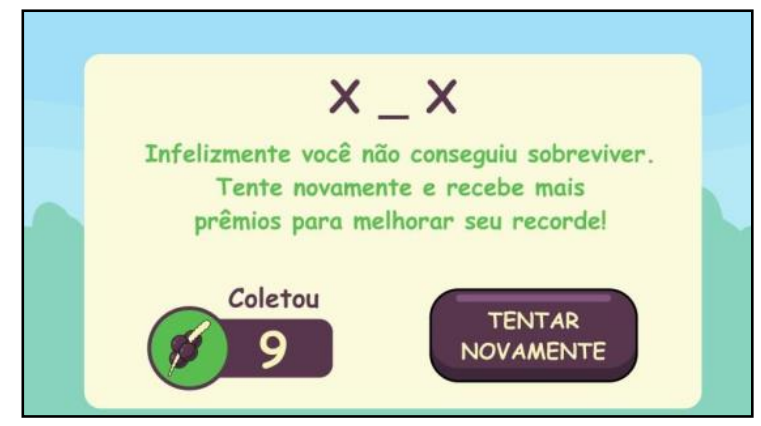

Figura 7. Tela de derrota

\section{Conclusão}

Este artigo propôs um jogo digital que visa melhorar o grau de conscientização e de informação da população sobre formas de prevenção da doença de Chagas, transmitida principalmente por alimentos mal higienizados, além das etapas de tratamento da doença, através das experiências adquiridas durante o jogo. É esperado que o jogo ofereça uma boa qualidade de aprendizagem, possuindo conteúdo embasado na teoria sobre a doença e aplicando-o ludicamente como forma de ensino para atingir desde o público infantil até o 
adulto. Além disso, desperta interesse do público em aprender devido à forma divertida e descontraída com que passa informação, demonstrando que o entretenimento é um aliado da educação para aprimorar os cuidados com a própria saúde.

Como trabalhos futuros, pretende-se primeiramente realizar testes de uso em larga escala. Nestes testes, serão usados questionários para os usuários sugerirem melhorias no jogo, para que assim, futuras versões proporcionem maior qualidade de experiência aos jogadores. Pretende-se ainda melhorar o realismo do jogo e os fatores de compartilhamento com redes sociais, tornando-o mais dinâmico e acessível para que os usuários tenham uma melhor experiência de aprendizado. Mas para um aplicativo desse porte ganhar destaque no mercado, é preciso ganhar apoio da população e de empresas, pois somente com isso será possível publicação e divulgação em larga escala para conquistar espaço de mercado e atingir um grande número, alcançando cada vez mais regiões do país onde a doença é uma enfermidade com taxa considerável de ocorrências.

\section{Referências}

Klaus Schwab (2016), The Fourth Industrial Revolution, Edipro, 1th edição.

Mizoguti, Isabela Lie (2018) "Doença de Chagas: A culpa é do Açaí?", Boletim Informativo: junho, 2018 v. 1, n. 1.

Rezende, J. M. (2017) “Clinical phases and forms of Chagas disease”, Federal University of Goias, Goias, Brazil

Falkembach, Gilse A. Morgental (2016) “Jogos educacionais", Universidade Federal do Rio Grande do Sul, Centro Interdisciplinar de Novas Tecnologias na Educação.

Panosso, Mariana G. (2015) “Características atribuídas a jogos educativos: uma interpretação Analítico-Comportamental" Revista Quadrimestral da Associação Brasileira de Psicologia Escolar e Educacional, SP. Volume 19, Número 2, maio/agosto de 2015: 233-241.

Pellegruine, Marina Joaquim (2007) "A importância dos jogos e das brincadeiras na educação infantil”, Pontifícia Universidade Católica de São Paulo, Faculdade de Educação.

Costa, Alanna Carla (2016) "Satisfação dos pacientes com doença de Chagas atendidos por um serviço de atenção farmacêutica no estado do Ceará, Brasil" Universidade Federal do Ceará, Instituto do Coração.

Ampudia, Ricardo (2018) "Celular é mais utilizado do que computador para acessar internet no Brasil” https:/www1.folha.uol.com.br/tec/2018/07/celular-e-mais-utilizadodo-que-computador-para-acessar-internet-no-brasil.html, julho.

Secretaria de Vigilância em Saúde (2012) "Doença de Chagas Aguda e distribuição espacial dos triatomíneos epidemiológicos”, Ministério da Saúde. Volume 50. jan. 2019

Ortega, Luciana V. Nunes (2016) "Jogos e brincadeiras no processo de ensino - Gestão Universitária” http://www.gestaouniversitaria.com.br/artigos-cientificos/jogos-ebrincadeiras-no-processo-de-ensino-aprendizagem-na-educacao-infantil, dezembro. 
Kaneto, L. Alves (2018) "Educational Workshop using games improves self-monitoring of blood glucose among children" Faculdade das Américas, Escola de Enfermagem, São Paulo, SP, Brazil.

Ministério da Saúde. (2019) "Doença de Chagas: o que é, causas, sintomas, tratamento e prevenção" http://portalms.saude.gov.br/saude-de-a-z/doenca-de-chagas, janeiro.

Agencia Brasil (2019) "Número de pessoas que tem celular aumenta 147\% em dez anos, diz IBGE" http://agenciabrasil.ebc.com.br/geral/noticia/2016-12/numero-de-pessoasque-tem-celular-aume nta-147-em-dez-anos-diz-ibge, janeiro.

Médicos sem fronteiras (2018), "Doença de Chagas" https://www.msf.org.br/o-quefazemos/atividades-medicas/doenca-de-chagas 